Cultures \& Conflits

18 | été 1995

La violence politique des enfants

\title{
"Bassidje", auxiliaires juvéniles de la révolution iranienne
}

Farhad Khosrokhavar

\section{(2) OpenEdition}

1 Journals

\section{Édition électronique}

URL : http://journals.openedition.org/conflits/465

DOI : $10.4000 /$ conflits.465

ISSN : $1777-5345$

Éditeur :

CCLS - Centre d'études sur les conflits lilberté et sécurité, L'Harmattan

\section{Édition imprimée}

Date de publication : 15 juillet 1995

ISSN : 1157-996X

\section{Référence électronique}

Farhad Khosrokhavar, " "Bassidje", auxiliaires juvéniles de la révolution iranienne », Cultures \& Conflits

[En ligne], 18 | été 1995, mis en ligne le 04 mars 2005, consulté le 30 mars 2021. URL : http://

journals.openedition.org/conflits/465; DOI : https://doi.org/10.4000/conflits.465

Ce document a été généré automatiquement le 30 mars 2021.

Creative Commons License 


\title{
"Bassidje", auxiliaires juvéniles de la révolution iranienne
}

\author{
Farhad Khosrokhavar
}

La définition de l'enfant en termes d'âge soulève de nombreux problèmes. Peut être considéré enfant celui que les adultes d'une société désignent comme tel ${ }^{1}$. En situation normale, les enfants sont dans une situation de minorité politique et civique par rapport aux adultes. On distingue généralement enfant, adolescent, adulte, mais ces distinctions sont souvent remises en cause en situation de crise. En plein bouillonnement révolutionnaire, certains groupes «d'enfants» (en réalité les adolescents et les post-adolescents) parviennent à s'affranchir provisoirement de la tutelle des parents et se constituent en "pré-adultes » (c'est-à-dire ayant la plupart des prérogatives des adultes) sous la tutelle d'autres adultes. Ceux-ci leur ouvrent un espace de pouvoir qui n'est pas explicitement défini en termes d'âge ou de génération, mais n'en demeure pas moins lié à leur situation de facto de jeunes mineurs. Dans cet espace se dessine la stratégie de groupes d'adultes qui entendent exploiter les jeunes comme un groupe de pression dans l'échiquier social et politique, au besoin en leur accordant certains privilèges dont les mineurs sont dépourvus. Ces avantages les constituent en pré-adultes. Parallèlement, chez les jeunes mineurs, cet espace est exploité dans le sens de leur promotion sociale, pour accélérer leur passage au statut «d'adulte avant la lettre » ainsi que pour satisfaire aux aspirations qu'une société souvent bloquée et dépourvue d'appareil politique adéquat ne parvient pas à réaliser. Les tenants du pouvoir manipulent la jeunesse qui n'a pas toujours les moyens critiques pour se distancier et se faire juge de la situation. Mais d'un autre côté, les jeunes se servent des nouveaux moyens mis à leur disposition par le nouveau pouvoir, pour faire avancer leurs revendications: acquérir un statut, du pouvoir ou même des moyens économiques au détriment des adultes «contre-révolutionnaires » ou sans allégeance révolutionnaire, et accéder à l'âge adulte avant l'échéance institutionnelle ou coutumière. Ainsi dans la révolution iranienne, des jeunes, âgés de quatorze et dix-huit ans, ont eu à exercer une part du pouvoir local, au niveau des Comités ${ }^{2}$, des organismes révolutionnaires ou de Bassidje ${ }^{3}$. L'espace de manipulation, en situation de crise, entre 
les détenteurs du pouvoir et les jeunes est ainsi assez flou, chacun tentant de tirer son épingle du jeu et de tirer avantage de la situation, marquée par la crise globale.

2 Pré-adultes, adultes, sur-adultes et crise de société Ainsi voit-on, dans le cas de la révolution iranienne, l'émergence politique d'une classe d'âge, disons adolescente, se réclamant d'un patriarche octogénaire pour contester la génération des pères, détentrice du pouvoir, justifier qu'on introduise les notions de sur-adultes, Khomeyni et ses émules, de pré-adultes investis d'un pouvoir pré-révolutionnaire et d'adultes, dévalués en « sous-adultes ", produits de la délégitimation des pères. La distinction préadulte, adulte, sur-adulte, définie en termes de légitimité remet provisoirement en cause la dichotomie majeur/mineur. Dans la révolution iranienne, dans deux périodes spécifiques, de durée inégale, la distinction majeur/mineur s'estompe et laisse la place au registre pré-adulte/adulte/sur-adulte ${ }^{4}$. Le jeune (majeur ou mineur) qui abonde dans le sens du nouveau pouvoir, se voit traiter de pré-adulte, tant que la gestation du pouvoir ou les besoins de la guerre le rendent indispensable au nouveau régime qui s'en sert, en l'occurrence, pour mater la société: les jeunes deviennent les agents de répression du nouvel ordre, le suivent " aveuglément ", mettant l'enthousiasme de leur jeunesse à son service. Ils le font parce que les liens sociaux traditionnels se sont relâchés du fait de la modernisation et qu'ils ne s'identifient plus comme par le passé à l'ordre communautaire, jadis porteur de valeurs et pourvoyeur d'emplois. A présent, un nouvel acteur social entre en jeu, dont le rôle s'est grandement accentué par sa mainmise sur la rente pétrolière, à savoir l'Etat. Celui-ci se réclame de la légitimité révolutionnaire de Khomeyni et surmonte ainsi, pour les jeunes gens qui y adhèrent, la déficience traditionnelle du pouvoir en légitimité. Désormais, il arrive, le temps d'une ardeur révolutionnaire, à convaincre les jeunes en voie de modernisation, de sa légitimité (il incarne l'islam authentique et révolutionnaire en la personne de Khomeyni). Simultanément, il lui est possible d'accéder à la condition adulte, au besoin en discréditant les adultes. Les deux périodes où les pré-adultes se voient accorder un privilège sont d'une part pendant la période de crise de quelques mois qui a précédé le renversement du Chah (février 1979) où les jeunes lycéens ont joué un certain rôle dans la fermeture des établissements scolaires et dans l'expansion des idéologies de gauche islamiques. Mais les jeunes (post-adolescents ou non) ont joué aussi un rôle majeur après la révolution, dans la période d'affrontement entre le régime islamiste et l'extrême-gauche d'une part, et dans la guerre ${ }^{5}$ contre le régime irakien d'autre part. L'un des lieux de recrutement des adolescents et des jeunes pré-adultes sont les « associations islamiques » qui sont nées, peu avant le renversement du Chah, dans les institutions scolaires. Après la révolution, elles sont progressivement purgées et prises en charge par le Hezbollah, le parti religieux quasi-unique, omnipotent.

Des lycéens pas comme les autres Tout au long de la crise contre l'extrême-gauche à partir de la deuxième année de la révolution et plus généralement, dans la période de guerre contre l'Irak (1980-88), opèrent au sein des « associations islamiques » des lycées de jeunes lycéens, qui jouent un rôle particulièrement répressif vis-à-vis de la société globale d'une part, vis-à-vis de leurs pairs, d'autre part. En sévissant contre les autres lycéens et même contre le corps enseignant (qu'ils peuvent accuser d'être contrerévolutionnaire ou " tiède " vis-à-vis de la révolution), ces jeunes lycéens assument des fonctions d'adultes et parviennent à surmonter les désavantages liés à leur condition de lycéens. L'Association islamique du Lycée est, dans la plupart des cas, composée de trois sections : 
- La Guidance (érchâd) : si un élève n'est pas « islamique » dans son comportement, s'il écoute trop de musique non-révolutionnaire ou occidentale, s'il ne manifeste pas assez d'ardeur révolutionnaire, on en "discute " avec lui et on cherche à le ramener sur le "droit chemin ». S'il continue, il sera sanctionné et sa famille risquera de se faire stigmatiser de contre-révolutionnaire.

- Renseignements (tadjasos) : ceux qui terminent l'enseignement secondaire et veulent entrer à l'université doivent avoir l'approbation de l'Association islamique du Lycée, qu'interrogent les agents du Renseignement du Ministère de la Culture et de l'Enseignement supérieur : fait-il ses prières quotidiennes, est-il bon « musulman » (i.e. défenseur du régime islamiste) ?; a-t-il des penchants répréhensibles sur le plan doctrinal (maktabi) ? N'a-t-il pas des sympathies pour les groupes d'extrême-gauche? Quelqu'un de sa famille est-il membre des groupes contre-révolutionnaires?

- Enfin, l'Unité de propagande (tablighât), qui a pour tâche de mettre des affiches au mur, organiser des manifestations les jours de défilés pour le régime, encourager le départ des jeunes lycéens sur le front, etc. L'Association islamique est autonome vis-àvis de la direction du lycée et dépend directement d'une section spéciale du Ministère de l'éducation nationale. Ses membres ont un pouvoir d'intimidation vis-à-vis de la hiérarchie scolaire et ne sont plus, dès lors, des élèves comme les autres. Leurs relations avec la section spéciale leur donnent un redoutable moyen de pression vis-àvis des enseignants qui les craignent et ne peuvent plus les noter normalement. Ils auront ainsi des bonnes notes, indépendamment de leurs performances scolaires, ce qui encourage les jeunes « cancres » à les rejoindre, ne serait-ce que pour se protéger d'une mauvaise notation. Il se crée un groupe de pression qui ne se soumet plus à la hiérarchie scolaire et s'en autonomise partiellement. L'association propose aux lycéens des avantages s'ils adhèrent à Bassidje et partent au front : ils seront généreusement notés, ils n'auront pas à assister aux cours fastidieux et surpeuplés du Lycée, ils pourront user de leur temps sur le front à leur guise, organiser leurs loisirs, surtout, apprendre à manier les armes et faire ainsi preuve de virilité, etc.

Une prédication efficace Des prédicateurs sont envoyés dans les lycées pour, dans le cadre de l'Association islamique, exhorter les jeunes à partir sur le front: «Vous êtes jeunes, vous êtes purs et non-souillés par le péché, vous pouvez aller au front; nous, nous sommes pécheurs, nous ne pouvons pas nous racheter par le martyre!» disait l'un de ces prédicateurs. Toute une idéologie de la pureté juvénile est utilisée par ces prédicateurs itinérants, pour attirer les jeunes dans l'organisation Bassidje : être jeune c'est le pouvoir d'accéder à la félicité éternelle, moyennant le martyre, ce qui n'est pas le cas pour les gens âgés, entachés de péché et dépourvus de cette pureté liliale qu'exige le martyre sensu eminenti. Les jeunes se voient promus acteurs préférentiels du martyre, en raison même de leur jeunesse, ce qui est un élément tout à fait nouveau dans l'idéologie islamique où l'âge accordait traditionnellement un privilège intrinsèque. Certes, la jeunesse a toujours bénéficié d'une incontestable faveur dans la religiosité populaire chi'ite où les enfants de l'imam Hoseyn ont souffert le martyre à Karbélâ. Il n'en demeure pas moins que leur martyre était célébré dans le cadre du sacrifice de Hoseyn qui occupait la place centrale. A présent, par un retournement lourd de sens, la jeunesse se fait le prédicat essentiel du martyre, les acteurs juvéniles étant promus au devant de la scène et la jeunesse n'étant plus exaltée comme un état subordonné au privilège de l'âge : Ali-Akbar et Ali-Asghar étaient avant tout les enfants de Hoseyn, pour la religiosité traditionnelle, lors même que le jeune Bassidji est luimême un Hoseyn en herbe, n'étant subordonné à aucune structure patriarcale 
préexistante. La jeunesse se voit ainsi accorder une place fondamentale dans la nouvelle religiosité. L'un des piliers de l'encadrement au sein de Bassidje, est la propagande idéologique. Le clergé préposé à cette tâche exhorte à la mort et, à cette fin, dévalorise systématiquement la vie. A l'occasion de la mort de 17 jeunes Bassidji sur le front le 16/12/1983, le cheikh Ansâriân fait un prône qui est distribué en cassettes dans les centres de Bassidje. Il y joue constamment sur le double registre de «boire la douce potion du martyre " (charbaté chiriné chahâdat râ nouchidan) et la potion sucrée (le thé sucré ou toute autre boisson sucrée) : le premier est une allégorie, le second, une réalité physique. Il incite l'audience à opter pour la première alternative, en raison de sa suavité éternelle, et de mourir "suavement" en martyr, au lieu de répéter ad nauseam l'acte monotone et animal de prendre son thé sucré au petitdéjeuner, tous les matins, aux seules fins de vivre une vie dénuée de sens : «Mon Dieu! De ces douces potions (de martyre) que Tu fais boire à ces jeunes, donne nous un peu ! C'est quoi cette vie? Tous les jours, prendre son petit-déjeuner, son déjeuner ;demain, à nouveau prendre son petit-déjeuner, son déjeuner; après-demain de nouveau, la répétition du même schéma : prendre son petit-déjeuner, son déjeuner (les larmes de l'audience se donnent à entendre dans l'enregistrement ). C'est cela la vie ? Nous en avons assez de ces potions du matin (sous-entendu : le thé sucré), donne-nous de cette autre potion (la potion du martyre). Celle-ci est fort douce, l'autre n'est rien en comparaison! Mélange d'eau et de sucre! Ce n'est pas cela l'humanité (âdamiyat), ce n'est pas cela être un homme digne de ce nom (ensâniyat)! (Donne-nous) de cette potion, de celle qui, jusqu'à l'éternité, dissout l'homme en Toi (Dieu), de cette potion, de celle qui, jusqu'à l'éternité, rend ivre de Toi ! C'est cela la vraie potion! ».

Martyropathes, jeunesse ludique, les déshérités Il n'existe pas un groupe unique de jeunes, adolescents ou post-adolescents dans le mouvement révolutionnaire iranien. Plusieurs types de jeunes sont en jeu. Pour commencer, Bassidje monnaye l'incapacité de vivre des jeunes post-adolescents qu'anime le rêve révolutionnaire, irréalisable dans la vie. Pendant la révolution ils étaient adolescents, à présent, post-adolescents, voire jeunes majeurs. Ce rêve n'est plus porteur de sens dans la société réelle qui ne veut plus entendre parler de révolution. En s'enfermant dans Bassidje, cette partie de la jeunesse (que l'on qualifiera de martyropathe) se sauve et de la société réelle et de la déception consécutive à l'évanouissement des rêves. Bassidje préserve le rêve révolutionnaire, de sa mutation en cauchemar; il prévient l'effondrement de l'identité éphémère de ses jeunes adhérents. D'où son succès considérable pendant les premières années où la révolution gardait encore son aura. Son plus grand succès, Bassidje l'emporte au moment crucial où l'épreuve du réel est encore ambivalente, dans les premières années de la révolution: le Guide de la révolution personnifie encore la révolution dans son utopie effervescente; et celle-ci se revigore momentanément du contraste entre une société, qui fait figure d'impure et la conscience acérée des martyrs, parce qu'il contredit ce rêve que les jeunes caressent malgré le démenti du réel. Bassidje sauve le jeune martyropathe en s'instituant instance de purification du corps social. Cette organisation se sacralise autant de la désorganisation sociale, qu'elle se fait instance purificatrice. Elle organise la purification par le passage à la mort. Mais si la mort purifie, l'organisation de la transition de la vie au trépas, au service du nouvel ordre purificateur, se fait par un processus où le sentiment de culpabilité du jeune individu est mis au service de sa propre répression et de sa sujétion au nouveau pouvoir despotique. Bassidje, par la mort qu'elle administre aux jeunes martyropathes, allège momentanément leur conscience, du poids du péché qui les accable, péché qui tient à 
leur conviction d'avoir involontairement trahi la révolution, puisqu'elle a échoué dans

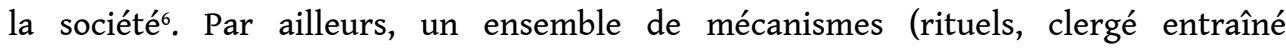
spécialement pour cette tâche, cours d'idéologie, mises en scènes théâtrales...) donnent aux jeunes (adolescents ou jeunes majeurs) le sentiment de la solidarité dans la mort, dans une atmosphère de fin du monde. On culpabilise les survivants de ne pas mourir alors que d'autres succombent sur le champ de bataille, on leur inculque l'indignité de la vie et l'inutilité de survivre dans un monde impie. Conditionnés, certains adolescents succombent au charme de cette idéologie mortifère. Bassidje encadre d'une autre manière encore, celle-là positive, les jeunes non-martyropathes qui la rejoignent pour autre chose que mourir. A ces chômeurs, elle offre un travail plus ou moins rémunéré7 Les jeunes plus ou moins dégagés des liens communautaires qui assignaient naguère un sens tout fait à la vie et un avenir professionnel plus ou moins pré-tracé, sont en quête d'un travail où ils puissent se réaliser pleinement, où l'autonomie économique et le sentiment de participer à la modernité aillent de pair. Le chômage est le déni de l'une ou de l'autre de ces exigences. Bassidje pare à ce manque. Elle enrôle les jeunes, remplit leur temps, les prend en charge, ne leur laisse aucun répit, leur procure la possibilité d'exercer leur créativité au péril de leur vie. Mille subterfuges, des trouvailles sans nombre jalonnent les combats où l'ingéniosité des jeunes Bassidji trouve un immense champ d'application. Ce besoin d'exercer ses talents dans une société qui, autrement, les frustre et où l'économie est au point mort, est bien relevé par ce jeune Bassidji, plusieurs fois blessé au front et qui dit au cours d'un entretien: Maintenant que je me suis spécialisé dans le déminage, je ne peux plus m'en passer. Ce pôle actif d'identité où l'ego se déploie de manière constructive (même si en l'occurrence, on se réalise dans la destruction), est ressenti très vivement par certains volontaires de Bassidje, postadolescents ou jeunes majeurs. On se "réalise " dans le maniement des mines tout comme, dans les sociétés modernes, on le fait en pianotant sur son ordinateur. Chez ces jeunes, ce n'est pas comme chez les martyropathes, la production de soi dans la mort, mais la production de soi au risque de la mort, dans une conjoncture qui ne leur laisse pas d'autres choix. Une autre catégorie de jeunes, formée surtout d'adolescents et de post-adolescents, rejoint Bassidje par besoin ludique. Ceux-ci, pour d'autres raisons que les martyropathes, refusent la quotidienneté morose de la post-révolution. Ils cherchent l'ivresse de la guerre et l'enthousiasme du combat qui font perpétuer l'effervescence révolutionnaire et l'unanimité des coeurs qui manquent cruellement dans la réalité post-révolutionnaire, jusque dans la famille Les adolescents sont, par ailleurs, comme possédés du fantasme de leur immortalité. Ils ne se sentent pas, à proprement parler immortels stricto sensu, mais ils ont la certitude de ne pas mourir en ce moment précis, dans cette période où l'espace qui s'ouvre entre l'enfance (surmontée) et l'âge adulte (non encore entièrement advenu) leur donne l'impression d'invulnérabilité. Ce fantasme d'éternité est exploité par le pouvoir islamiste sous une forme spécifique, liée au martyre : "Même si tu meurs, tu seras immortel, tu feras partie des martyrs; eux, ils ne meurent pas!" Bassidje est tout au long de la guerre, l'abondance au superlatif; on y mange relativement bien, il n'y a pas de pénurie (contrairement à l'armée traditionnelle qui n'est pas aussi bien approvisionnée), on a accès aux divertissements (les films sur magnétoscopes sont nombreux, souvent peu censurés), et surtout on fraternise ensemble dans une communauté close que soude la vie en groupe sur le champ de bataille. Bassidje, pour les jeunes démunis, est aussi, souvent, une promotion onirique, dans un ensemble clos qui ne connait pas les déchirements de la société réelle, dans une communauté à huis clos dotée d'une image 
d'Epinal et soustraite aux dures réalités économiques de la société. Il existe une dernière catégorie de jeunes Bassidji, ceux qui s'enrôlent dans cette organisation malgré eux. Quelquefois, les parents prennent eux-mêmes l'initiative d'envoyer leur fils au Bassidje : soit parce qu'ainsi ils évitent l'envoi des autres fils sur le front; soit parce qu'ils sont sous pression autant de la part de l'Etat que des familles des voisins dont les fils se trouvent sur le front; dans ce cas, refuser de les imiter est considéré comme une entorse aux lois de bon voisinage et comme l'expression d'un intolérable égoïsme: le partage du sacrifice familial est l'une des expressions de l'identité du groupe. Ce fait est particulièrement visible dans les petites villes où la structure communautaire n'est pas aussi entamée que dans les grandes unités urbaines; soit pour donner une légitimité aux revendications des familles aux avantages clientélistes de l'Etat; soit enfin quelquefois, parce que dans les couches les plus démunies, le départ $\mathrm{du}$ fils au front allège les dépenses de famille où on a une bouche de moins à nourrir. Dans ce cas, la famille se console en espérant que le fils ne sera pas tué sur le front et qu'ainsi, elle pourra gagner sur plusieurs (avantages matériels et clientélistes ainsi que la survie du fils). Ces cas-là sont fortement minoritaires. Les cas dominants sont ceux où l'Etat parvient à attirer les jeunes dans ses rets en faisant miroiter à leurs yeux la gloire guerrière, le prestige de l'islam ou le ludisme intense d'une fête où ils accèdent à la condition de pré-adulte, au grand dam de la famille qui ne dispose pas d'autres moyens de pression, sinon morale ou affective. Il se constitue une identité « jeune » au sein du Bassidje, où les distinctions entre adolescence, post-adolescence et l'âge adulte (jeunes majeurs) ont tendance à s'estomper. Les jeunes s'appuient sur l'Etat révolutionnaire répressif pour s'affranchir de la tradition, tant l'évolution de la famille leur semble souvent lente et insuffisante. Mais simultanément, le ralliement à cet Etat n'est pas sans engendrer des tensions : il isole les jeunes qui collaborent avec le nouvel ordre répressif, il les fait honnir de la société, les accule à chercher appui dans cet Etat total à qui ils font acte d'allégeance, ne serait-ce que parce qu'ils se sentent stigmatisés dans une société hostile qui les perçoit comme les agents répressifs d'un ordre exécré. La multitude d'organisations répressives qui pullulent après la révolution permet à une partie de la jeunesse (adolescents ou majeurs) d'y adhérer et d'y investir cette part de leur identité qui n'arrive plus à s'investir dans la société, vu la déstructuration de l'ordre communautaire, mais aussi, vu le refus dont ils font implicitement l'objet dans la société, par leur adhésion à l'Etat islamiste. Les organismes de répression (le Comité, l'Armée des pasdarans, la Croisade pour la construction, la Croisade universitaire ...) quadrillent la société mais ils pourvoient aussi la jeunesse hezbollah d'un sentiment d'identité, en institutionnalisant son attachement affectif à Khomeyni. dans une société en crise économique, secouée par la guerre et en butte à une révolution qui désorganise tout, la remise en cause des entraves de la famille signifie ipso facto pour les jeunes, leur adhésion quasi-forcée à l'Etat, tant pour des raisons affectives que matérielles et clientélistes, aucun autre levier social n'étant disponible pour assurer leur insertion, ou leur promotion économique, aucun autre principe d'identification ne se présentant à leur psyché déstructurée dans un champ culturel (artistique) et économique qui leur échappe.

6 L'Unité des jeunes Bassidji contre la société : la sincérité répressive Bassidje engendre intra muros l'Unités , dans une société déchirée par la désunion à peine deux années après la période de la bouillonnante unanimité révolutionnaire. L'unité de Bassidje, répressive pour le reste de la société n'est pas un vain mot. Sous le pouvoir islamique, toute forme d'expression libre est perçue comme une entorse à l'unité 
imposée par le Hezbollah. Mais le mythe de l'unité au sein de Bassidje n'opère pas sous une forme purement symbolique. Elle se veut en acte, l'unité des âges. A la différence de la société où la rupture entre la jeunesse et les aînés est devenue une évidence primordiale, au sein de Bassidje les jeunes, voire les adolescents, luttent dans l'union des coeurs, aux côtés des vieillards. Ceux-ci travaillent souvent dans les diverses sections de la Croisade pour la Construction, cette autre organisation révolutionnaire spécialisée dans le développement des zones rurales qui s'est occupée, tout au long de la guerre, des problèmes de logistique et de construction des routes et des ponts, au service de l'Armée des pasdarans ${ }^{9}$ où de bas en haut de la hiérarchie des âges les membres vivent sur un mode mythiquement unitaire. Les clivages d'une société où, du fait de la modernisation, jeunes et vieux habitent deux univers mentaux hétérogènes, ont disparu. L'éclatement social entre eux est occulté par l'idéologie mortifère qui donne aux uns et aux autres le fantasme de l'unité organique. Jeunes et vieux se réconcilient dans l'envoûtement d'une mort unifiante. Une néo-mystique nécrophile les cimente dans la poursuite de cette mort qui leur prescrit une vocation commune. Bassidje incarne aussi l'unité dans l'abondance pour les adolescents en quête d'aventure et de ludisme. La néo-communauté Bassidji n'est pas hantée par cette pénurie qui guette la société réelle dans sa vie quotidienne. On y mange à sa faim et même fort bien, tout y est gratis pro deo, on y cultive une économie de festivité pure, fondée sur la démonétisation des relations d'échange et d'acquisition des denrées. Le bazar, les industries, l'Etat et presque toutes les institutions économiques font à Bassidje des « dons » (souvent forcés) qui bannissent toute carence en son sein, au plus fort de la guerre, dans cette période où la rareté et l'appauvrissement sont un fait d'évidence dans la société. En Bassidje se réalise l'unité dans l'égalité. La hiérarchie économique étant bannie de Bassidje, la hiérarchie de décision étant intériorisée et les chefs étant désignés dans l'ensemble selon une procédure méritocratique aux niveaux moyens et inférieur (les plus chevronnés sont à la tête des divers groupes), la discipline militaire étant peu respectée, il existe une atmosphère propice à l'éclosion d'une grande fraternité d'armes. A divers échelons on perçoit, à la différence de l'armée, une grande cordialité et une indéniable convivialité. Par exemple, dans l'armée, pour obtenir une permission, il suffit souvent d'acheter l'assentiment vénal de ses supérieurs. Le volontaire de Bassidje n'a pas besoin de tels expédients. Souvent il refuse de partir en permission pour demeurer auprès de ses compagnons d'armes. De même, dans l'armée, un soldat qui n'en peut se tire une balle dans la jambe (on l'appelle Khozani : tirer sur soi) pour se faire hospitaliser ${ }^{10} \mathrm{Ce}$ genre de phénomène est rarissime dans Bassidje où, à l'inverse, la tendance est de prendre à la légère les blessures et même, après un séjour à l'hôpital pour blessure grave, de retourner, avant le terme de sa convalescence, sur le front. Plus l'unité sociale autour du rêve de l'unanimité se disloque et plus l'exigence de l'unité devient impérative chez le martyropathe ; et plus il porte à l'ordre répressif une affection illimitée, pour imposer ladite unité à la société, malgré elle. Son idéal unitaire ne peut se réaliser pleinement qu'au sein de Bassidje, pas dans la société réelle. Il se rend bien compte que malgré la répression, la société refuse d'abdiquer la vie et que la volonté de vivre est incommensurablement plus puissante que celle de mourir. Ceci la lui fait prendre en horreur. Pour la punir du péché qui consiste à vouloir vivre, il lui dénie la majorité politique. Seuls sont habilités à prendre part au champ politique les purs qui ont renoncé à vivre. Ceux qui tiennent par-dessus tout à la vie sont par définition indignes de participer à la politique et doivent être destitués de leur citoyenneté. La répression au nom du Pur, par le pouvoir hezbollah, 
remplit cet office. L'aspiration à mourir implique ainsi la répression de ceux qui veulent vivre. Le despotisme du Pur est fondé sur le primat de la mort et sur le déni de légitimité à ceux qui sont animés du désir de vivre. D'un côté, le jeune, féru de mort impose le despotisme aux vivants; de l'autre, il opte pour la mort, non pas dans la société, mais hors d'elle, dans cette néo-communauté de Bassidje qui est, en l'occurrence, une contre-société. La mort s'inscrit ainsi dans un destin qui a sa cohérence interne: elle est l'issue fatale d'une situation désespérée où les jeunes désespérés optent pour la mort, tout en réprimant ceux qui souscrivent à la vie. La volonté unitaire des jeunes martyrs est à analyser dans une double perspective : c'est le constat de la rupture de l'unité dans la société (c'est la fin de l'unanimité dans le mouvement révolutionnaire); mais c'est aussi la volonté de perpétuer l'unité brisée, par le haut, par l'appel à la mort, par le despotisme du Guide qui exhorte à la mort purificatrice et coalescente. Cela n'est possible que parce que la rupture est consommée entre la société réelle et ces révolutionnaires attardés que sont les martyrs. Plus ils font l'éloge de l'unité, plus ils se fondent dans l'unité de Bassidje, et plus cette unité mythique se construit contre la société réelle qui a répudié la révolution et son mythe fondateur de l'unité. Le martyropathe parcourt le chemin inverse de la société réelle : plus celle-ci se désidentifie du pouvoir hezbollah et le rejette, et plus celui-ci s'y identifie et s'accroche fébrilement à un régime honni de la majorité. Ce phénomène est particulièrement visible dans la notion de sincérité chez eux. Ce qui différencie leur sincérité de l'état d'esprit général, est leur incompréhension du devenir effectif de la révolution. Le mouvement révolutionnaire a évolué ; son utopie flamboyante a cédé la place à une amère désillusion; de moins en moins les gens croient en elle et de plus en plus ils s'en détachent, "lui tournent le dos» (expression fréquente dans les conversations $\left.{ }^{11}\right)$. C'est cette histoire de la désintégration progressive du mouvement révolutionnaire que les martyropathes se refusent à admettre. ils sont en décalage par rapport au reste de la société, ils n'arrivent pas à percevoir l'usure de l'utopie, son érosion face au démenti de la réalité. Les martyropathes refusent fondamentalement l'histoire. Leur refus est motivé par leur incapacité à s'adapter à la réalité nouvelle. Ce travail est l'histoire de ceux qui refusent l'histoire et l'historicité des mouvements sociaux. Ils en sont restés à la vision des commencements du mouvement révolutionnaire et refusent d'en reconnaitre la désagrégation irréversible.

7 Conclusion La crise révolutionnaire, l'émergence d'un nouveau pouvoir répressif qui a à lutter contre de nombreux groupes radicalisés qui cherchent l'appropriation du champ politique, sont à l'origine de l'enrôlement des jeunes et en particulier des adolescents et des post-adolescents par le nouveau pouvoir. Celui-ci s'en sert pour réprimer la société, mais aussi pour trouver de la chair à canon dans le champ de bataille. Ces jeunes (adolescents, post-adolescents ou jeunes majeurs), ne forment pas un groupe homogène. Ils sont soit férus de mourir (les martyropathes), soit en quête de se nourrir dans une conjoncture économique difficile (déshérités), soit à la recherche du jeu et du divertissement (la jeunesse ludique). Ils se laissent mobiliser par le nouveau pouvoir parce qu'ils y trouvent partiellement leur compte : ils passent plus rapidement l'épreuve de transition à l'âge adulte; ils trompent leur ennui dans une société où la morosité liée au puritanisme révolutionnaire restreint dans ses fêtes et ses jouissances; ils quittent l'enceinte scolaire et se dirigent sur le champ de bataille; enfin, ils renversent, pour un temps, la hiérarchie où ils étaient en bas de l'échelle. La jeunesse populaire, celle qui se qualifie de déshéritée, adhère souvent à Bassidje pour faciliter sa promotion sociale sur le front, ou après, dans l'appareil d'Etat. Cela étant, 
plus le nouveau pouvoir se raffermit et moins la capacité d'auto-affirmation des jeunes trouve à s'exprimer. Ils perdent une grande partie de leur autonomie de facto du début de la révolution, suite à l'éclatement du pouvoir. Ils sont progressivement exclus des domaines où ils pouvaient intervenir au début ; enfin, avec l'affermissement du régime et la fin de la guerre, ils perdent les restes des privilèges qui leur étaient laissés tout au long de la guerre, au sein de Bassidje. De pré-adultes, les adolescents se retrouvent dans une situation où leurs sont déniés les droits des adultes -à moins d'avoir suffisamment grandi entre-temps pour prétendre aux privilèges liés à leur nouvel condition adulte dans une structure du pouvoir où leur allégeance au nouvel ordre répressif leur donne le « droit » d'occuper un poste dans l'appareil d'Etat.

\section{NOTES}

1. Cet article est l'abrégé d'une partie d'un ouvrage à paraître en 1995, L'Islamisme et la mort, le martyre révolutionnaire en Iran, Les éditions l'Harmattan.

2. Le Comité (Komitéh en persan) a été l'échelon de base du pouvoir local, pendant la crise du régime impérial. Après la révolution les innombrables comités qui avaient foisonné pendant la révolution sont progressivement dans le nouvel Etat. Dans ces comités, les jeunes ont joué un rôle majeur.

3. Bassidje est l'organisation créée peu après le renversement du Chah par le nouveau pouvoir révolutionnaire pour mettre la jeunesse populaire au service de la révolution. Bassidje a été par la suite intégrée au sein de l'armée des Pasdaran, autre organisation militaire qui a vu le jour après la révolution pour la protéger contre les monarchistes et qui s'est constituée en force armée indépendante du corps de l'armée traditionnelle. Dans la guerre contre l'Irak, Bassidje, forte de presque un demi-million d'adhérents, encadrait des recrues volontaires. Le jeune Bassidji (celui qui adhère à Bassidje), entre une douzaine et une vingtaine d'années (avec une minorité de vieillards), s'est fait remarquer par son dévouement total et son absence de crainte face à la mort. Il a souvent servi dans la stratégie de chair à canon, contre l'armée irakienne. Cf. pour une description de ces acteurs mortifères Farhad Khorsokhavar, "Chiisme mortifère, le combattant de la foi", L'Homme et la Société, n 107-108, 1993 ; "Le Pur et l'Impur", Peuples Méditerranéens, n 50, 1990 ; W. Scmunker, "Iranische Märtyrertestamente", Die Welt des Islams, 27, $\mathrm{n}^{\circ}$ 4, 1987.

4. Peuvent être traités de sur-adultes tous ceux qui sont dotés d'un charisme ou d'un prestige tel que les jeunes majeurs ou mineurs, sont prêts à les suivre et ce faisant, se rehausser de leur prestige. Dans la révolution iranienne, mis à part Khomeyni, il y a eu quelques figures de proue comme Taléghâni ou quelques leaders d'extrême gauche pour leurs tenants, qui ont bénéficié de ce prestige.

5. Cf. pour le récit purement descriptif des jeunes prisonniers iraniens en Irak Ian Brown, Khomeyni's forgotten sons, The story of Iran's boy soldiers, Grey Seal Books, London, 1990.

6. Ces faits sont développés dans L'islamisme et la mort, voir note 1. 
7. Les jeunes qui en font la demande sont payés comme un petit fonctionnaire du secteur public.

8. Au début de la révolution, la thématique majeure est la liberté (le slogan fondamental des manifestants est "liberté, indépendance, république islamique"). L'Unité n'est qu'une notion stratégique pour maintenir l'unanimité contre le régime du chah. Après la révolution, l'Unité empiète sur la liberté et se la subordonne. Le Hezbollah combat les tenants de la liberté au nom de l'unité islamique. Cf. Le rêve impossible, l'anthropologie de la révolution iranienne, à paraître

9. Cf. le quotidien Keyhan section spéciale, le 31.6.1366 (1987). On est de plain-pied dans une néo-communauté[[Contrairement à la communauté traditionnelle dont l'identité se définit par des relations de parenté et de proximité, distincte de celle du pouvoir, Bassidje unifie des jeunes qui trouvent une nouvelle communauté dans leur rapport au sacré et au pouvoir qui émane, selon eux, de l'islam, en particulier de Khomeyni. Cette nouvelle forme d'agrégation sociale n'est pas que la reproduction de la communauté traditionnelle, même si elle en garde certains traits. nous l'appellerons néocommunauté .En son sein, contrairement à la communauté traditionnelle, les frontières s'estompent avec le pouvoir et il règne une atmosphère effervescente et égalitaire qui la distinguent de l'ordre communautaire stricto sensu.

10. Les services de renseignement de l'armée y regardant de plus près, les soldats mettent quelquefois une planche de bois sur leur jambe avant de tirer, afin de stimuler la balle à distance.

11. Pour toutes ces expressions cf. notre thèse d'Etat, op cit., annexe, les entretiens.

\section{RÉSUMÉS}

Organisation créée peu après le renversement du Chah par le nouveau pouvoir révolutionnaire pour mettre la jeunesse populaire au service de la révolution, Bassidje a été par la suite intégrée au sein de l'armée des Pasdarans, autre organisation militaire qui a vu le jour après la révolution pour la protéger contre les monarchistes et qui s'est constituée en force armée indépendante du corps de l'armée traditionnelle. Dans la guerre contre l'Irak, Bassidje, forte de presque un demimillion d'adhérents, encadrait des recrues volontaires. Le jeune Bassidji (celui qui adhère à Bassidje), entre une douzaine et une vingtaine d'années s'est fait remarquer par son dévouement total et son absence de crainte face à la mort. Il a souvent servi de chair à canon, contre l'armée irakienne. Bassidje monnayait l'incapacité de vivre des jeunes post-adolescents qu'anime le rêve révolutionnaire, irréalisable dans la vie. En s'enfermant dans Bassidje, cette partie de la jeunesse « martyropathe » se sauvait de la société réelle et de la déception consécutive à l'évanouissement des rêves. Bassidje préservait le rêve révolutionnaire, de sa mutation en cauchemar, il prévenait l'effondrement de l'identité éphémère de ses jeunes adhérents. D'où son succès considérable pendant les premières années où la révolution gardait encore son aura. 
INDEX

Thèmes : Bassidje, Pasdaran

Index géographique : Iran

Mots-clés : enfants, forces paramilitaires, guerre, kamikaze (missions suicide), Militaires, répression, violence 